\title{
Controlled Production of Unsupported Lipid Bilayers As Supports For Electron Microscopy of Single Membrane Particles
}

\author{
Mike Strauss, David E. McAlduff, F. Peter Ottensmeyer \\ Ontario Cancer Institute and Department of Medical Biophysics, University of Toronto, 610 \\ University Avenue, Toronto, ON, M5G 2M9, Canada \\ Previous work in the lab saw the successful formation of lipid monolayers on the surface of \\ water in a Petri dish, and the subsequent bilayer formation upon vertical dipping of an electron \\ microscope grid through the surface of the monolayer. The copper grid, which was covered with \\ carbon stabilized fenestrated plastic, was coated with the monolayer on each side in this process \\ such that the holes in the plastic allow bilayer formation $[1,2]$.
}

Bilayers were indeed formed. However, the monolayer formation was not necessarily of equal lipid density each time. Moreover, the manual dipping required a steady hand. The results of the process proved to be usable, but somewhat inconsistent, with variable bilayer coverage of the holes, up to $80 \%$. In addition, while the mass/density of the bilayer films was consistent within a given batch of grids made from the same monolayer, a variation of up to $25 \%$ was observed between batches from different monolayers.

To improve on the consistency, a Langmuir-Blodgett trough has been purchased (Nima Technology, Coventry, England) with a pressure sensor, compression barriers, and an automated dipping mechanism specifically designed for electron microscopy grids held in forceps. This device permits a controlled compression of the lipid monolayer, with a concomitant measurement of the surface tension of the layer. Thus different lipid surface densities can be achieved, from sparse coverage to arrangements in which the lipid heads are neatly arrayed, with hydrophobic tails pointing away from the water subphase in a close-packed assembly. Signal feedback from the pressure sensor is relayed to movable barriers to yield monolayers of constant surface tension, even while dipping. The device is equipped with a computer controlled stepper motor with a variable dipping speed. This permits a smooth entry of the grid into the subphase, which is essential to prevent the monolayer from tearing or buckling as it adheres to the moving grid.

A continuation of the dipping motion then deposits the bilayer-containing grid into a submersed grid box on the bottom of the trough for storage before subsequent use. As before [2], proteins can then be incorporated into the freshly generated bilayers. This has been done by fusing a proteoliposome with the bilayer using a salt gradient. Successful fusions have been obtained using lipid compositions (PC/PS:1/1) which were similar for both the proteoliposomes and the lipid bilayers.

[1] D.E. McAlduff, Master's Thesis, University of Toronto, (2000)

[2] D.E. McAlduff, F.P. Ottensmeyer, J. Microscopy (in press). 


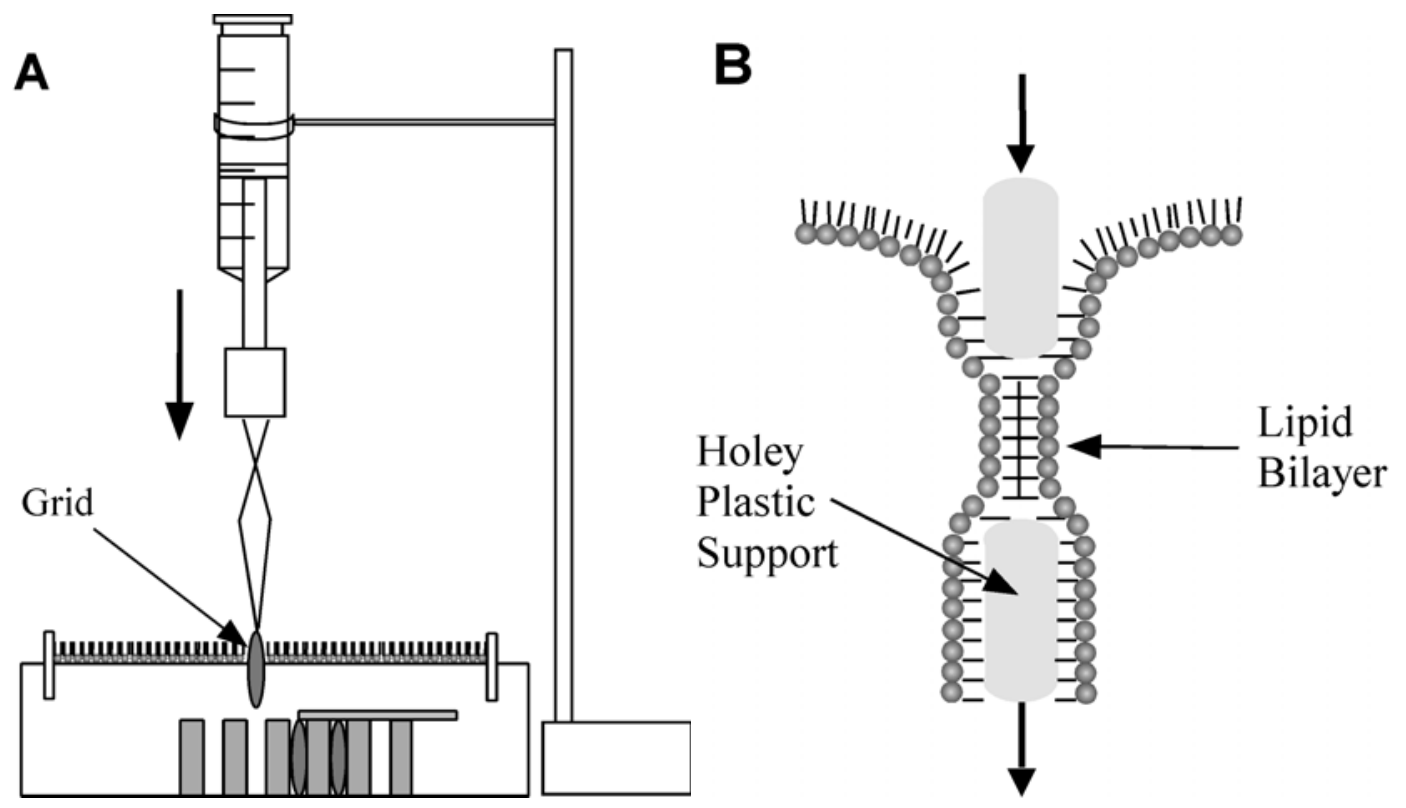

Figure A: Schematic of the dipping mechanism previously used.

Figure B: Formation of the lipid bilayer over a hole in the plastic support.

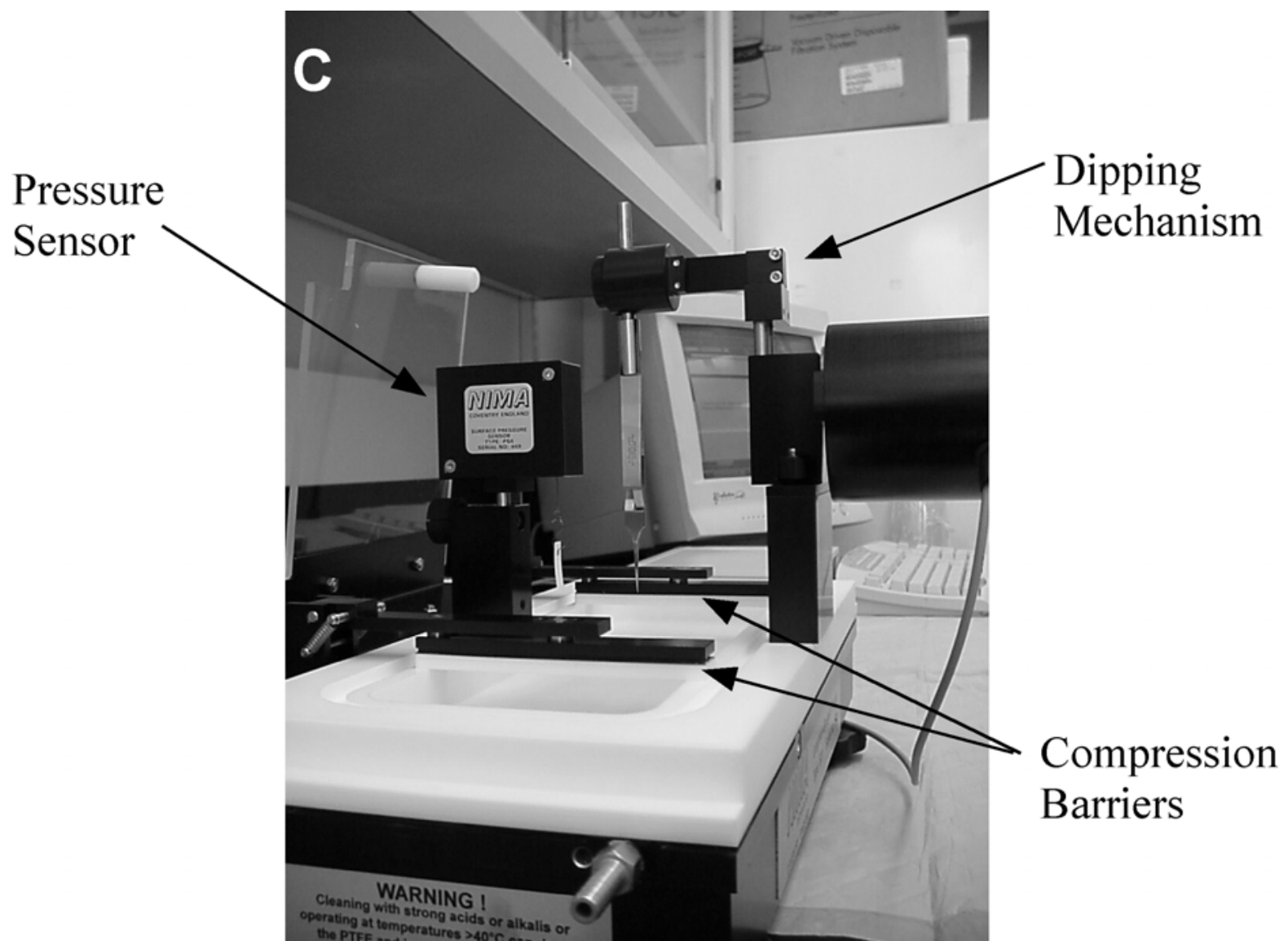

Figure C: Langmuir-Blodgett trough. 